to deny that the addition of pure air to already existing sanitary services is most desirable.

Dr. Brend advocates as a remedy the expansion or rarefaction of our large towns and the segregation of our factories (a substantial piece of work for the proposed Ministry of Health !). Perhaps in practice the difficulties of removing smoke from existing towns is less than that of removing our populations to better sites. We have sewage systems in all our large towns, and the problem of removing waste products of combustion may be no greater than that of getting rid of the waste products of the body or of the wash-tub.

The demand for a Ministry of Health, the permanent staff of which "must consist almost exclusively of medical and scientific men," has much to recommend it, but we are rendered somewhat dubious of the practical results of their lucubrations when we find the author remarking of vaccination: "It is open to argument whether this precaution is still essential purely as a prophylactic, though it is of course important during an epidemic among persons brought in contact with the disease." We wonder whether Dr. Brend has considered the practical steps requisite to vaccinate persons brought in contact with the disease when, for instance, a tramp suffering from modified small-pox has spread the disease in perhaps half a dozen different towns. Nor are we clear as to the author's reasons for considering that the diagnosis of this disease has improved whilst opportunities of observing it have become enormously lessened.

As a whole the volume is well worthy of study. A wide range of subjects affecting public health is discussed-from the sale of abortefacients to the public health duties of the Treasury.

\section{OPTICAL THEORIES.}

Optical Theories, Based on Lectures delivered before the Calcutta University. By Dr. D. N. Mallik. Pp. r81. (Cambridge: At the University Press, I9I7.) Price 7s. 6d. net.

THE subject of physical optics has undergone ranked as one of the great divisions of mathematical science; now it has become almost wholly absorbed by a sister science. The phenomena of optics, by their variety and ever-increasing practical importance, attract and deserve specialised study; but the underlying theory can no longer be studied apart from electricity, and the long succession of theories of the æther in the nineteenth century form a closed chapter in the history of science. There seems little likelihood that the chapter will be reopened. In these circumstances the best approach to the subject may be a matter of doubt, depending a great deal on the temperament of the student. Those who are historically minded will urge that the present position is best apprehended by fol-

NO. 2501 , VOL. IOO] lowing the steps which have led to it; others will consider that adherence to the traditional mode of approach tends to root in the mind an obsolete mode of thought, and it is better not to trifle with the freedom which is now offered. Dr. Mallik's book offers a compromise which should be acceptable to both sides. A survey of the more essential properties and differences of the mechanical æthers that were once proposed, subordinated to the purpose of illustrating the difficulities which the electromagnetic theory strides over so easily that they pass almost unnoticed; afterwards, an account of the modern theory and results, contrasted where necessary with the mechanical æethers-these seem to bring out the essential aspects of our present knowledge, without undue neglect of the lessons of the past.

Dr. Mallik divides his subject into four principal chapters : early theories, elastic solid theory, electromagnetic theory, and electron theory. The separation of the two last has some disadvantages. So far as it relates to free æther, the electromagnetic theory is independent of electrons; but in the early extensions to dielectric media the object presumably was to evade, rather than to theorise on, the relations of matter to ather. To make a full discussion of the mechanical implications of this approximate treatment seems unnecessary at the present day; the undeveloped notions of permeability and specific inductive capacity scarcely need to be taken so seriously. An excellent summary of the whole argument is given in the concluding chapter, which shows how far we have travelled since speculations on the optical medium first began. The theory of relativity and the quantum theory are not included in the scope of the book.

The volume is written for fairly advanced students, and the discussion necessarily is mainly mathematical. A great amount of work is surveyed in brief compass; and most readers will find fresh information, and arguments that are new to them. According to the author's plan, only those developments are treated which afford a means of discriminating between rival theories; and the student will do well to follow his guidance through the bewildering mass of investigations which still confront the learner, as well as through the débris of wrecked hypotheses.

\section{OUR BOOKSHELF.}

\section{Chile. Pp. 3or. (Santiago: The Chilean} Government, 1915.)

THIS anonymous volume written in English is doubtless meant to diffuse a knowledge of Chile and particularly of Chilean resources and trade in English-speaking countries, but there is no preface to indicate its aim or the personality of its editor. In about fifty short chapters it contains a comprehensive survey of Chile, its life and conditions, including a good deal of statistical 